\title{
Synthesis, in vitro anticancer activity evaluation and docking investigations of novel aromatic chalcones
}

\author{
Vankadari Srinivasarao, Chaturvedula Radha Krishna, Macha Ramesh, Tigulla Parthasarathy* \\ Department of chemistry, University College of Science, Osmania University, Saifabad, Hyderabad, India
}

Email address:

sarathychem@gmail.com (T. Parthasarathy)

\section{To cite this article:}

Vankadari Srinivasarao, Chaturvedula Radha Krishna, Macha Ramesh, Tigulla Parthasarathy. Synthesis, in vitro Anticancer Activity Evaluation and Docking Investigations of Novel Aromatic Chalcones, Modern Chemistry. Vol. 1, No. 1, 2013, pp. 1-7. doi: $10.11648 /$ j.mc.20130101.11

\begin{abstract}
In a wide search program toward new anticancer agents, a series of Aromatic chalcones have been synthesized by condensing benzaldehyde derivatives with arylmethylcetone in potassium hydroxide ethanol according to the ClaisenSchmidth condensation at room temperature. The synthetic chalcones have been determined by IR spectroscopy and $1 \mathrm{H}-$ NMR spectroscopy. The anticancer activitiesof the compounds were evaluated against in vitro using human tumour cell lines of Jurkat and HL-60 cell lines and MTT assay. From the series, two compounds $(4,15)$ exhibited potent growth inhibitory effects against the proliferation of human T-lymphocyte leukemia compared to the parent unsubstitutedchalcone. The result shows that the electron donating groups moiety may increase anticancer activity. Aromatic chalcones with hydroxyl group,methoxy group on A ring at positions 2 or 3 are considered as lead compounds for generation of new potential anticancer drugs in future.Similarly,the compounds $(7,12)$ exhibited potent growth inhibitory effects against HL-60 cells. The results are very encouraging. Future studies include testing the compounds in vivo with and without radiation. Docking studies with 1NKP have shown that the compound 17 has highest IC50 against human leukemia cells (HL-60).
\end{abstract}

Keywords: Aromatic Chalcones Synthesis, Anticancer Activity, Docking Studies, Mtt Assay

\section{Introduction}

At present, there is a growing interest in the discovery of new anticancer agents to bate against inhibition of MCF-7 human breast cancer cells growth[1], inhibition of cyclooxygenase-2 in RAW264.7 cells[2] and inhibition of iNOS protein expression.Chalcones(1,3-diphenyl-propene-1one) pertaining to the flavanoid family both natural as well as synthetic products have been revised for their wide biological activities such as anticancer [3-9] antiinvasive[10] antituberculosi[11] antifungal[12], antibacterial[13], antitmour[14],antiinflammator[15], antioxidant[16].Studies on the bioavailability of Aromatic chalcones from natural sources are limited, but synthetic Aromatic chalcones have been reported to have a wide range of biological properties, especially anticancer ,antibacterial[17-19] and antifungal activities[20].In an effort to develop potent anticancer agents a series of Aromatic chalcones we have reported the process synthesis of Aromatic chalcones as well as their inhibitory activity against the human tumour cell lines of
Jurkat and HL-60. Many efforts are being made to treat different types of cancer over the past few decades.Cancer is treated either by removing the excess growth or radiation therapy or by chemotherapy. The last alternative, i.e., the chemotherapy is gaining increasing attention due to ease of administration. To facilitate the chemotherapy, there is a relentless march to invent new drugs. In this thrust, molecules with alkenone moiety as integral part of the molecule hold potential in the treatment of cancer. In the light of this,chalcones hold promise as a class of natural molecules bearing anticancer activity.Earlierinvestigations have revealed that 2'-oxygenated chalcone derivatives have been shown to possess a wide variety of activities like prostaglandin E2 production inhibition in rat peritoneal macrophages [21].Recent studies have thrown light on some 2'aminochalcone derivatives which exhibit antitumor activity[22] and 2-hydroxy chalcones are reportedas inhibitors of peroxynitrile-mediated oxidation of low-density lipoproteins (LDL) [23] and also to possess antileishmanial activity [24].Furthermore, cytotoxic effects of 2'-oxygenated chalcone derivatives on human tumor cells such as Jurkat and 
novel pyrimidine fused indazole derivatives on U937 cell lines wasreported.The present article throws light on the synthesis of specific chalcones, their interactions on acute T-cell leukemia (Jurkat) and human leukemia (HL-60) cell lines.The data is further compared with docking studies with the crystal structure of (1NKP) protein.

\section{Methods and Materials}

\subsection{Synthesis}

Reagents and solvents were purchased from commercial sources and used without further purification unless otherwise specified. Air-and moisture-sensitive liquids and solutionswere transferred via syringe or stainless steel cannula.Organic solutions were concentrated by rotary evaporation below 450c at approximately $20 \mathrm{~mm}$ $\mathrm{Hg}$.Allnonaqueous reactions were carried out under anhydrous conditions using flame-dried glassware within an argon atmosphere in dry freshly distilled solvents, unless otherwise noted.Yields referred to chromatographical separation, unless other-wise stated. Reactions were monitored by thin-layer chromatography carried out on $0.15-0.20 \mathrm{~mm}$ silica gel plates(MERCK company)using UV light as the visualizing agent. Chromatography was performed on Merck silica gel 60F254 aluminum plates mesh,(Merck separation materials pvt company limited) using petroleum ether and acetone as the eluanting solvent. 1H-NMR spectra were determined on a Bruker Ultra shield 500 spectrometers using tetramethylsilane(TMS) as an internal reference.IR spectra were recorded with a FT Perkin Elmer 16 $\mathrm{PC}$ spectrometer on $\mathrm{KBr}$ disks. Chemical shifts were recorded in parts per million downfield from tetramethylsilane(TMS).J-values were given in $\operatorname{Hertz}(\mathrm{Hz})$.Abbreviationsused were singlet(s), doublet(d), triplet(t), quartet(q), broad(b), and multiplet(m). The cooled solution of the different substituted acetophenone, aromatic aldehyde in ethanolic solution treated with potassium hydroxide solution, undergoing condensation forms different Aromatic chalcones. 1H NMR, IR spectra were consistent with the assigned structures. Compounds(1-19) were synthesized different Aromatic chalcones with different substituents on Aromatic rings A,B(Table 1).

Table 1. Structure and analytical data of the chalcone analogues.

\begin{tabular}{|c|c|c|c|c|c|c|c|c|}
\hline No. & $\mathbf{R}_{1}$ & $\mathbf{R}_{2}$ & $\mathbf{R}_{\mathbf{3}} \mathbf{R}_{\mathbf{4}}$ & $\mathbf{R}_{5}$ & $\mathbf{R}_{6}$ & $\mathbf{R}_{7}$ & $\mathbf{R}_{8}$ & Formula Yield (\%) \\
\hline 1 & $\mathrm{OH}$ & $\mathrm{OMe}$ & $\mathrm{OMeH}$ & $\mathrm{H}$ & $\mathrm{Cl}$ & $\mathrm{H}$ & $\mathrm{H}$ & $\mathrm{C}_{17} \mathrm{H}_{15} \mathrm{ClO}_{4} 70$ \\
\hline 2 & $\mathrm{OH}$ & $\mathrm{OMe}$ & $\mathrm{OMeH}$ & $\mathrm{H}$ & $\mathrm{F}$ & $\mathrm{H}$ & $\mathrm{H}$ & $\mathrm{C}_{17} \mathrm{H}_{15} \mathrm{FO}_{4} 87$ \\
\hline 3 & $\mathrm{OH}$ & $\mathrm{OMe}$ & $\mathrm{OMeH}$ & $\mathrm{H}$ & $\mathrm{H}$ & $\mathrm{H}$ & $\mathrm{Cl}$ & $\mathrm{C}_{17} \mathrm{H}_{15} \mathrm{ClO}_{4} 89$ \\
\hline 4 & $\mathrm{OMe}$ & $\mathrm{OMe}$ & $\mathrm{OMeH}$ & $\mathrm{H}$ & $\mathrm{H}$ & $\mathrm{H}$ & $\mathrm{Cl}$ & $\mathrm{C}_{18} \mathrm{H}_{17} \mathrm{ClO}_{4} 67$ \\
\hline 5 & $\mathrm{OMe}$ & $\mathrm{OMe}$ & $\mathrm{OMeH}$ & $\mathrm{H}$ & $\mathrm{OMe}$ & $\mathrm{H}$ & $\mathrm{H}$ & $\mathrm{C}_{19} \mathrm{H}_{20} \mathrm{O}_{5} \quad 43$ \\
\hline
\end{tabular}

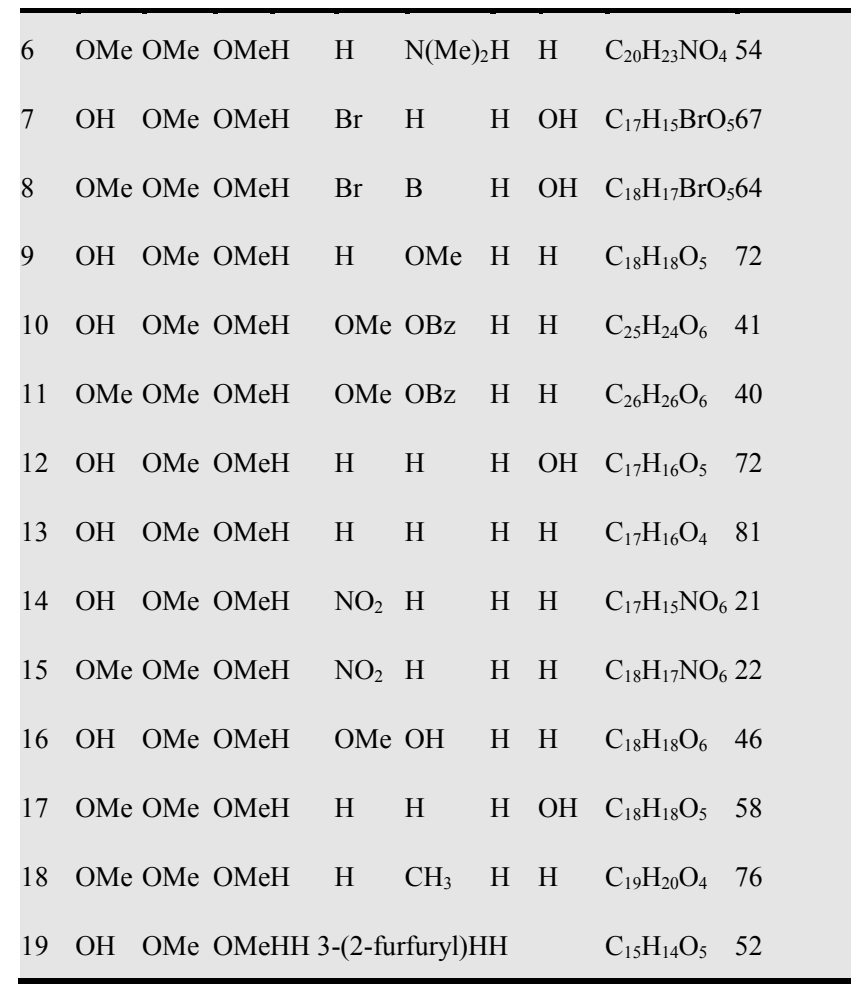

\subsection{ExperimentsGeneral Synthetic Method of Aromatic chalcones}

The melting points $(\mathrm{mp})$ were taken in open capillary tubes using Galenkampt apparatus and are uncorrected. The IR spectra were recorded on Schimadzu FTIR 8201PC Spectrophotometers.1H-NMR spectra were determined on a Bruker Ultra shield 500 spectrometers using tetramethylsilane (TMS) as an internal reference.All the starting materials are commercially available. A number of known and novel (1-19)Aromatic chalcone derivatives were prepared by a Claisen-Schmidth condensation[25] of the appropriate aldehyde and acetophenone derivatives(scheme 1).General procedure for the synthesis of chalcones: a solution of substituted acetophenone $(5 \mathrm{mM})$ and aromatic aldehyde $(5 \mathrm{mM})$ in ethanol $(15 \mathrm{mM})$ was cooled to $5-100 \mathrm{c}$ in a nice bath. The cooled solution was treated with adding a small portion of pulverized potassium hydrox$\operatorname{ide}(10 \mathrm{mM})$. The reaction mixture was magnetically stirred for 60 minutes and then left overnight or longer, monitored by thin layer chromatography using developing solvent $n$ hexane-acetone $(5: 1)$. The resulting dark solution was diluted with ice water and carefully acidified using dilute hydrochloric acid.Thechalcone which separated as a yellow solid was collected by filtration after washing with water and further purified by crystallization from methanol. The residues were purified and the yield of chalcones was 45$87 \%$. The structures of the chalcones were characterized by IR and $1 \mathrm{H}$ NMR spectral data. 


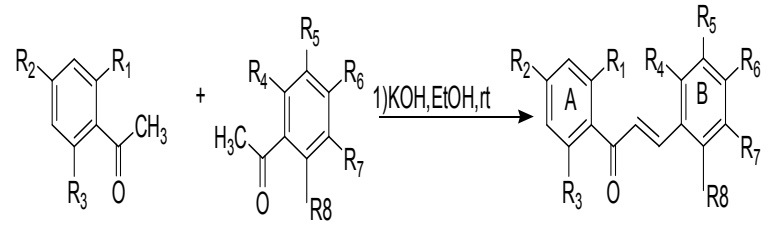

Scheme 1. Claisen-Schimidt condensation,(1)KOH(1.5eq),substituted acetophenone(1eq), substituted benzaldehyde(1 eq);EtOH, room temperature; (2) $10 \% \mathrm{HCl}$ solution.<smiles>[R]c1cc([R])c(C(=O)/C=C/c2c([R6])c([R6])c([R6])c([R6])c2[R])c([R])c1</smiles>

Compound 1: (E)-3-(4-chlorophenyl)-1-(2-hydroxy4,6-dimethoxyphenyl)prop-2-en-1-one

This compound is synthesized using2-hydroxy-4,6dimethoxyacetophenone (1.96g.) and4-chlorobenzaldehyde (1.4g.) as starting materials. IR $(\mathrm{KBr}) 1630(\mathrm{C}=\mathrm{O}), 1566$ $(\mathrm{C}=\mathrm{C}) \mathrm{cm}^{-1} .{ }^{1} \mathrm{H}$ NMR $\left(\mathrm{CDCl}_{3}\right), \partial 14.23(\mathrm{~s}, 1 \mathrm{H}), 7.87(\mathrm{~d}, 1 \mathrm{H}$ $\mathrm{J}=15.52 \mathrm{~Hz}), 7.71(\mathrm{~d}, 1 \mathrm{H} \mathrm{J}=15.52 \mathrm{~Hz}), 7.53(\mathrm{~d}, 2 \mathrm{H}, \mathrm{J}=$ $8.49 \mathrm{~Hz}), 7.37(\mathrm{~d}, 2 \mathrm{H}, \mathrm{J}=8.49 \mathrm{~Hz}), 6.11(\mathrm{~d}, 1 \mathrm{H} \mathrm{J}=2.26$ $\mathrm{Hz}), 5.96(\mathrm{~d}, 1 \mathrm{H} \mathrm{J}=2.26 \mathrm{~Hz}), 3.91(\mathrm{~s}, 3 \mathrm{H}), 3.84(\mathrm{~s}$, $3 \mathrm{H})$.Yield $=70 \% ; \mathrm{mp}=173-175^{\circ} \mathrm{C} ;$ Anal. Calcd.for $\mathrm{C}_{17} \mathrm{H}_{15} \mathrm{ClO}_{4}$ :C, 64.06,H,4.74;found:C, 64.26; H, 4.69.

Compound 2: (E)-3-(4-fluorophenyl)-1-(2-hydroxy-4, 6-dimethoxyphenyl) prop-2-en-1-one

This compound is synthesized using2-hydroxy-4,6dimethoxyacetophenone (1.96g.) and4-fluorobenzaldehyde (1.24g.) as starting materials.IR(KBr)1632 $(\mathrm{C}=\mathrm{O}), 1572$ $(\mathrm{C}=\mathrm{C}) \mathrm{cm}^{-1} .{ }^{1} \mathrm{H}$ NMR $\left(\mathrm{CDCl}_{3}\right), \partial 14.19(\mathrm{~s}, 1 \mathrm{H}), 7.51-7.78(\mathrm{~m}$, $3 \mathrm{H}), 7.02-7.31(\mathrm{~m}, 3 \mathrm{H}) 6.08(\mathrm{~d}, 1 \mathrm{H} \mathrm{J}=2.22 \mathrm{~Hz}), 5.98(\mathrm{~d}, 1 \mathrm{H}$ $\mathrm{J}=2.22 \mathrm{~Hz}), 3.97(\mathrm{~s}, 3 \mathrm{H}), 3.81(\mathrm{~s}, 3 \mathrm{H})$.Yield $=87 \% ; \mathrm{mp}=$ 140-141 ${ }^{0} \mathrm{C}$;Anal.

Calcd.for

$\mathrm{C}_{17} \mathrm{H}_{15} \mathrm{FO}_{4}$ :,67.54;H,5.00;found:C, 67.50; H, 5.04.

Compound 3: (E)-3-(2-chlorophenyl)-1-(2-hydroxy4,6-dimethoxyphenyl)prop-2-en-1-one

This compound is synthesized using2-hydroxy-4,6dimethoxyacetophenone (1.96g.) and2-chlorobenzaldehyde (1.4g.) as starting materials.IR $(\mathrm{KBr}) 1630(\mathrm{C}=\mathrm{O}), 1556$ $(\mathrm{C}=\mathrm{C}) \mathrm{cm}^{-1} .{ }^{1} \mathrm{H}$ NMR $\left(\mathrm{CDCl}_{3}\right), 214.12(\mathrm{~s}, 1 \mathrm{H}), 8.18(\mathrm{~d}, 1 \mathrm{H} \mathrm{J}=$ $15.67 \mathrm{~Hz}), 7.81(\mathrm{~d}, 1 \mathrm{H} \quad \mathrm{J}=15.67 \mathrm{~Hz}), 7.20-7.69(\mathrm{~m}$, $4 \mathrm{H}), 6.08(\mathrm{~d}, 1 \mathrm{H} \mathrm{J}=2.36 \mathrm{~Hz}), 5.88(\mathrm{~d}, 1 \mathrm{H} \mathrm{J}=2.36 \mathrm{~Hz}), 3.91(\mathrm{~s}$, $3 \mathrm{H}), 3.82(\mathrm{~s}, 3 \mathrm{H})$.Yield $=89 \%$; $\mathrm{mp}=136-137^{\circ} \mathrm{C}$; Anal. Calcd.for

$\mathrm{C}_{17} \mathrm{H}_{15} \mathrm{ClO}_{4}$ :C,64.06;H,4.74;found:C,63.87; H,4.80.

Compound 4: (E)-3-(2-chlorophenyl)-1-(2,4,6trimethoxyphenyl)prop-2-en-1-one

This compound is synthesized using 2,4,6trimethoxyacetophenone $(2.10 \mathrm{~g}$.) and2-chlorobenzaldehyde (1.4g.) as starting materials.IR $(\mathrm{KBr}) 1632(\mathrm{C}=\mathrm{O}), 1560$ $(\mathrm{C}=\mathrm{C}) \mathrm{cm}^{-1} .{ }^{1} \mathrm{H}$ NMR $\left(\mathrm{CDCl}_{3}\right), 27.76(\mathrm{~d}, 1 \mathrm{H} \mathrm{J}=16.20$ $\mathrm{Hz}), 7.2-7.4(\mathrm{~m}, 4 \mathrm{H}), 6.85(\mathrm{~d}, 1 \mathrm{H} \mathrm{J}=16.20 \mathrm{~Hz}), 6.15$ (d, J = $2.33 \mathrm{~Hz}), 3.88(\mathrm{~s}, 3 \mathrm{H}), 3.78(\mathrm{~s}, 3 \mathrm{H})$.Yield $=67 \% ; \mathrm{mp}=111-$ $112^{\circ} \mathrm{C}$;Anal. Calcd.for $\quad \mathrm{C}_{18} \mathrm{H}_{17} \mathrm{ClO}_{4}: \mathrm{C}, 64.90 ; \mathrm{H}$, 5.10;found:C, 64.98;H,5.22.
Compound 5: (E)-1-(2,4,6-trimethoxyphenyl)-3-(4methoxyphenyl)prop-2-en-1-one

This compound is synthesized using 2,4,6trimethoxyacetophenone (2.10g.) and 4methoxybenzaldehyde1.36g.) as starting materials.IR (KBr) $1636(\mathrm{C}=\mathrm{O}), 1586(\mathrm{C}=\mathrm{C}) \mathrm{cm}^{-1} .{ }^{1} \mathrm{H}$ NMR $\left(\mathrm{CDCl}_{3}\right), \partial 7.56$ (m 2H,).7.41 (s 1H,),7.25(d, 2H, J = 8.27 Hz), 6.22(d, 2H J $=2.26 \mathrm{~Hz}), 3.9(\mathrm{~s}, 3 \mathrm{H}), 3.7(\mathrm{~s}, 6 \mathrm{H})$. Yield $=43 \% ; \mathrm{mp}=120$ $121^{0} \mathrm{C}$; Anal. Calcd.for ${ }_{19} \mathrm{H}_{20} \mathrm{O}_{5}: \mathrm{C}, 69.43 ; \mathrm{H}, 6.09$;found:C, $70.06 ; \mathrm{H}, 6.03$.

Compound 6: (E)-3-(4-(dimethylamino)phenyl)-1(2,4,6-trimethoxyphenyl)prop-2-en-1-one

This compound is synthesized using2,4,6trimethoxyacetophenone (2.10g.), and 4dimethylaminobenzaldehyde $(1.49$ g.) as starting materials.IR (KBr) $1624(\mathrm{C}=\mathrm{O}), 1588(\mathrm{C}=\mathrm{C}) \mathrm{cm}^{-1} .{ }^{1} \mathrm{H}$ NMR $\left(\mathrm{CDCl}_{3}\right), 27.38(\mathrm{~d}, 1 \mathrm{H}, \mathrm{J}=16.10 \mathrm{~Hz}), 6.58(\mathrm{~d}, 1 \mathrm{H}, \mathrm{J}=16.10$ $\mathrm{Hz}), 7.2(\mathrm{~d}, 2 \mathrm{H} \mathrm{J}=1.88 \mathrm{~Hz}), 6.68(\mathrm{~d}, 2 \mathrm{H}, \mathrm{J}=1.88 \mathrm{~Hz}), 6.08(\mathrm{~d}$, $2 \mathrm{H} \mathrm{J}=2.32 \mathrm{~Hz}$ ), 3.7-3.85(s, 9H),2.98(s, 6H). Yield = 54\%; $\mathrm{mp}=149-150^{\circ} \mathrm{C}$; Anal. Calcd for $\mathrm{C}_{20} \mathrm{H}_{23} \mathrm{NO}_{4}: \mathrm{C}$, 70.29;H,6.73;found:C,70.03;H,6.87.

Compound 7: (E)-3-(5-bromo-2-hydroxyphenyl)-1-(2hydroxy-4,6-dimethoxyphenyl)prop-2-en-1-one

This compound is synthesized using2-hydroxy-4,6dimethoxyacetophenone (1.96g.) and 2-hydroxy-5bromobenzaldehyde(2.01g.) as starting materials.IR $(\mathrm{KBr}) 1638(\mathrm{C}=\mathrm{O}), 1584(\mathrm{C}=\mathrm{C}) \mathrm{cm}-1.1 \mathrm{H}$ NMR $(\mathrm{CDCl} 3)$, 214.30(s, 1H),9.7(s, 1H),7.9(d, 2H, J = 8.36 Hz), 7.6(s, 1H), $7.2(\mathrm{~d}, 1 \mathrm{H}, \mathrm{J}=16.2 \mathrm{~Hz}), 6.8(\mathrm{~d}, 1 \mathrm{H}, \mathrm{J}=16.2 \mathrm{~Hz}), 5.9(\mathrm{~d}, 1 \mathrm{H} \mathrm{J}$ $=8.26 \mathrm{~Hz}), 3.9(\mathrm{~s}, 3 \mathrm{H}), 3.8(\mathrm{~s}, 3 \mathrm{H})$. Yield $=67 \%$; $\mathrm{mp}=177-$ 1780C;Anal.

Calcd.for

C17H15BrO5:C,53.79; H,3.95;found:C,54.02'H, 3.87.

Compound 8: (E)-3,(4,5-dibromo-2-hydroxyphenyl)1-(2,4,6trimethoxyphenyl)prop-2-en-1-one

This compound is synthesized using 2,4,6trimethoxyacetophenone (2.10g.)and 2-hydroxy-5bromobenzaldehyde(2.01g.) as starting materials. IR (KBr) $1630(\mathrm{C}=\mathrm{C}), 1580(\mathrm{C}=\mathrm{O}) \mathrm{cm}^{-1} .{ }^{1} \mathrm{H}$ NMR (DMSO), $210.18(\mathrm{~s}$, $1 \mathrm{H}), 7.56(\mathrm{~m}, 2 \mathrm{H}), 7.41(\mathrm{~s}, 1 \mathrm{H}), 7.25(\mathrm{~d}, 2 \mathrm{H} \mathrm{J}=8.27 \mathrm{~Hz}), 6.2(\mathrm{~d}$, $2 \mathrm{H} \mathrm{J}=2.26 \mathrm{~Hz}), 3.9(\mathrm{~s}, 3 \mathrm{H}), 3.7(\mathrm{~s}, 6 \mathrm{H})$. Yield $=64 \% ; \mathrm{mp}=$ 164-165 ${ }^{\circ}$; Anal. Calcd.forC ${ }_{18} \mathrm{H}_{17} \mathrm{BrO}_{5}: \mathrm{C}, 54.93$; $\mathrm{H}$, 4.32;found:C,54.72;H,4.46;

Compound 9: dimethoxyphenyl)-3-(4-methoxyphenyl)prop-2-en-1-one

This compound is synthesized using2-hydroxy-4,6dimethoxyacetophenone (1.96g.) and4methoxybenzaldehyde $(1.36 \mathrm{~g})$ as starting materials.IR $(\mathrm{KBr}) \quad 1630 \quad(\mathrm{C}=\mathrm{O}), \quad 1584 \quad(\mathrm{C}=\mathrm{C}) \quad \mathrm{cm}^{-1} .{ }^{1} \mathrm{H} \quad \mathrm{NMR}$ $\left(\mathrm{CDCl}_{3}\right), 214.40(\mathrm{~s}, 1 \mathrm{H}), 7.9(\mathrm{~d}, 2 \mathrm{H} \mathrm{J}=8.26 \mathrm{~Hz}), 7.6(\mathrm{~d}, 2 \mathrm{H} \mathrm{J}=$ $8.26 \mathrm{~Hz}), 7.7(\mathrm{~d}, 1 \mathrm{H} \mathrm{J}=16.32 \mathrm{~Hz}), 6.97(\mathrm{~d}, 1 \mathrm{H} \mathrm{J}=16.32$ $\mathrm{Hz}), 6.1(\mathrm{~d}, 1 \mathrm{H} \mathrm{J}=2.36 \mathrm{~Hz}), 5.9(\mathrm{~d}, 1 \mathrm{H} \mathrm{J}=2.36 \mathrm{~Hz}), 3.9(\mathrm{~s}$, $3 \mathrm{H}), 3.8(\mathrm{~s}, 6 \mathrm{H})$.Yield $=72 \% ; \mathrm{mp}=109-110^{\circ} \mathrm{C}$; Anal. Calcd.for $\mathrm{C}_{18} \mathrm{H}_{18} \mathrm{O}_{5}: \mathrm{C}, 68.72 ; \mathrm{H}, 5.72$;found:C,69.12; H,5. 34.

Compound 10: 4-((E)-3-(2-hydroxy-4,6dimethoxyphenyl)-3-oxoprop-1-enyl)-2-methoxyphenyl benzoate

This compound is synthesized using 2-hydroxy- 
4,6dimethoxyacetophenone (1.96g.) and 4-benzyloxy-3methoxybenzaldehyde (2.42 g.) as starting materials.IR $(\mathrm{KBr}) \quad 1624 \quad(\mathrm{C}=\mathrm{O}), 1582 \quad(\mathrm{C}=\mathrm{C}) \quad \mathrm{cm}^{-1} .{ }^{1} \mathrm{H} \quad \mathrm{NMR}$ $\left(\mathrm{CDCl}_{3}\right), 26.89-7.80(\mathrm{~m}, \quad 10 \mathrm{H}), 6.11(\mathrm{~d}, \quad 1 \mathrm{H} \quad \mathrm{J}=2.36$ $\mathrm{Hz}), 5.90(\mathrm{~d}, 1 \mathrm{H} \mathrm{J}=2.36 \mathrm{~Hz}), 5.21(\mathrm{~s}, 2 \mathrm{H}), 3.94(\mathrm{~s}, 6 \mathrm{H}), 3.82(\mathrm{~s}$, $3 \mathrm{H})$.Yield $=41 \% ; \mathrm{mp}=123-124^{\circ} \mathrm{C}$; Anal. Calcd.for $\mathrm{C}_{25} \mathrm{H}_{24} \mathrm{O}_{6}: \mathrm{C}, 71.34 ; \mathrm{H}, 5.70$;found:C, 72.11; H,5.23.

Compound 11: 2-methoxy-4-((E)-3-(2,4,6trimethoxyphenyl)-3-oxoprop-1-enyl)phenyl benzoate

This compound is synthesized using 2,4,6trimethoxyacetophenone (2.10g.)and 4-benzyloxy-3methoxybenzaldehyde (2.42 g.) as starting materials.IR $(\mathrm{KBr}) 1626(\mathrm{C}=\mathrm{O}) ; 1580(\mathrm{C}=\mathrm{C}) \mathrm{cm}^{-1} .{ }^{1} \mathrm{H}$ NMR $\left(\mathrm{CDCl}_{3}\right), \partial$ $7.40-6.83(\mathrm{~m}, 10 \mathrm{H}), 6.15(\mathrm{~d}, 2 \mathrm{H} \mathrm{J}=2.26), 5.19(\mathrm{~s}, 2 \mathrm{H}), 3.95(\mathrm{~s}$, $3 \mathrm{H}), 3.85(\mathrm{~s}, 3 \mathrm{H}), 3.76(\mathrm{~s}, 6 \mathrm{H})$. Yield $=40 \% ; \mathrm{mp}=116-117^{0} \mathrm{C}$; Anal. Calcd.for $\mathrm{C}_{26} \mathrm{H}_{26} \mathrm{O}_{6}: \mathrm{C}, \quad 71.80 ; \mathrm{H}, 5.98$ :found:C, $72.42 ; \mathrm{H}, 5.72$;

Compound 12: (E)-1-(2-hydroxy-4,6dimethoxyphenyl)-3-(2-hydroxyphenyl)prop-2-en-1-one

This compound is synthesized using2-hydroxy-4,6dimethoxyacetophenone (1.96g.) and2-hydroxy benzaldehyde (1.22g.) as starting materials.IR (KBr)1640 $(\mathrm{C}=\mathrm{O}), 1560(\mathrm{C}=\mathrm{C}) \quad \mathrm{cm}^{-1} .{ }^{1} \mathrm{H} \quad \mathrm{NMR} \quad\left(\mathrm{CDCl}_{3}\right), \partial \quad 14.30(\mathrm{~s}$, $1 \mathrm{H}), 9.62(\mathrm{~s}, 1 \mathrm{H}), 8.1(\mathrm{~d}, 1 \mathrm{H} \mathrm{J}=16 \mathrm{~Hz}), 7.9(\mathrm{~d}, 1 \mathrm{H} \mathrm{J}=16$ $\mathrm{Hz}), 7.5(\mathrm{~d}, 1 \mathrm{H} \mathrm{J}=2.26 \mathrm{~Hz}), 7.13(\mathrm{~d}, 1 \mathrm{H} \mathrm{J}=2.26 \mathrm{~Hz}), 6.82(\mathrm{~m}$, $2 \mathrm{H}), 6.10(\mathrm{~d}, 1 \mathrm{H} \mathrm{J}=2.32 \mathrm{~Hz}), 5.9(\mathrm{~d}, 1 \mathrm{H} \mathrm{J}=2.32 \mathrm{~Hz}), 3.90(\mathrm{~s}$, $3 \mathrm{H}), 3.78(\mathrm{~s}, 3 \mathrm{H})$. Yield $=72 \% ; \mathrm{mp}=159-160^{\circ} \mathrm{C}$; Anal. Calcd.for $\quad \mathrm{C}_{17} \mathrm{H}_{16} \mathrm{O}_{5} \mathrm{z}: \mathrm{C}, 68.00 ; \mathrm{H}, 5.33$;found:C, 67.68;H,5.52.

Compound dimethoxyphenyl)-3-phenylprop-2-en-1-one

This compound is synthesized using2-hydroxy-4,6dimethoxyacetophenone (1.96g.) and benzaldehyde (1.06g.) as starting materials.IR $(\mathrm{KBr}) 1628(\mathrm{C}=\mathrm{O}), 1586(\mathrm{C}=\mathrm{C}) \mathrm{cm}^{-}$ ${ }^{1}{ }^{1} \mathrm{H}$ NMR $\left(\mathrm{CDCl}_{3}\right), \partial 14.20(\mathrm{~s}, 1 \mathrm{H}), 7.92(\mathrm{~d}, 1 \mathrm{HJ}=15.34$ $\mathrm{Hz}), 7.78(\mathrm{~d}, 1 \mathrm{HJ}=15.34 \mathrm{~Hz}), 7.5(\mathrm{~d}, 2 \mathrm{H} \mathrm{J}=8.16 \mathrm{~Hz}), 7.38(\mathrm{~m}$, $3 \mathrm{H}), 6.12(\mathrm{~d}, 1 \mathrm{H} \mathrm{J}=2.36 \mathrm{~Hz}), 5.98(\mathrm{~d}, 1 \mathrm{H} \mathrm{J}=2.36 \mathrm{~Hz}), 3.90(\mathrm{~s}$, $3 \mathrm{H}), 8.81(\mathrm{~s}, 3 \mathrm{H})$.Yield $=81 \% ; \mathrm{mp}=89-90^{\circ} \mathrm{C}$; Anal. Calcd.for $\mathrm{C}_{17} \mathrm{H}_{16} \mathrm{O}_{4}: \mathrm{C}, 71.83 ; \mathrm{H}, 5.63$;found:C,71.62; H,5.74;

Compound 14: (E)-1-(2-hydroxy-4,6dimethoxyphenyl)-3-(3-nitrophenyl)prop-2-en-1-one

This compound is synthesized using2-hydroxy-4,6dimethoxyacetophenone (1.96g.) and 2-nitrobenzaldehyde (1.51g.) as starting materials.IR (KBr)1640 $(\mathrm{C}=\mathrm{O}), 1580(\mathrm{C}=\mathrm{C}) \mathrm{cm}^{-1} .{ }^{1} \mathrm{H} \quad \mathrm{NMR} \quad\left(\mathrm{CDCl}_{3}\right), 214.05(\mathrm{~s}$, $1 \mathrm{H}), 8.46(\mathrm{~s}, 1 \mathrm{H}), 8.22(\mathrm{~d}, 1 \mathrm{H} \mathrm{J}=7.75 \mathrm{~Hz}), 7.98(\mathrm{~d}, 1 \mathrm{H} \mathrm{J}=$ $15.68 \mathrm{~Hz}), 7.74(\mathrm{~d}, 1 \mathrm{H} \mathrm{J}=15.68 \mathrm{~Hz}), 7.84-7.78(\mathrm{~m}, 2 \mathrm{H})$, $6.12(\mathrm{~s}, 1 \mathrm{H}), 5.98(\mathrm{~s}, 1 \mathrm{H}), 3.94(\mathrm{~s}, 3 \mathrm{H}), 3.85(\mathrm{~s}, 3 \mathrm{H})$. Yield $=$ $21 \%$; $\mathrm{mp}=169-170^{\circ} \mathrm{C}$;Anal. Calcd.for $\mathrm{C}_{17} \mathrm{H}_{15} \mathrm{NO}_{6}: \mathrm{C}, 62.00 ; \mathrm{H}, 4.59$;found:C, 61.98; H, 4.56.

Compound 15: (E)-1-(2,4,6-trimethoxyphenyl)-3-(3nitrophenyl)prop-2-en-1-one

This compound is synthesized using 2,4,6trimethoxyacetophenone $(2.10 \mathrm{~g}$.) and 2-nitrobenzaldehyde (1.51g.) as starting materials.IR $(\mathrm{KBr}) 1642(\mathrm{C}=\mathrm{C}) \mathrm{cm}^{-1} .{ }^{1} \mathrm{H}$ $\operatorname{NMR}\left(\mathrm{CDCl}_{3}\right), 28.70(\mathrm{~s}, 1 \mathrm{H}), 8.21(\mathrm{~d}, 1 \mathrm{H} \mathrm{J}=8.32 \mathrm{~Hz}), 7.82(\mathrm{~d}$,
$1 \mathrm{H} \mathrm{J}=8.32 \mathrm{~Hz}), 7.58(\mathrm{~m}, 1 \mathrm{H}), 7.42(\mathrm{~d}, 1 \mathrm{H} \mathrm{J}=16.2 \mathrm{~Hz}), 7.05$ $\mathrm{d}, \quad 1 \mathrm{H} \mathrm{J}=16.2 \mathrm{~Hz}), 6.18(\mathrm{~s}, 2 \mathrm{H}), 3.85(\mathrm{~s}, 3 \mathrm{H}), 3.75(\mathrm{~s}$, $6 \mathrm{H})$.Yield $=22 \% ; \mathrm{mp}=157-158^{\circ} \mathrm{C} ;$ Anal. Calcd.forC ${ }_{18} \mathrm{H}_{17} \mathrm{NO}_{6}: \mathrm{C}, \quad 62.97 ; \mathrm{H}, 4.95$;found:C, 62.78;H4.89.

Compound 16: (E)-3-(4-hydroxy-3-methoxyphenyl)1-(2-hydroxy-4,6-dimethoxyphenyl)prop-2-en-1-one

This compound is synthesized using 2-hydroxy-4,6'dimethoxyacetophenone (1.96g.) and 3-methoxy-4hydroxybenzaldehyde (1.52g.) as starting materials.IR

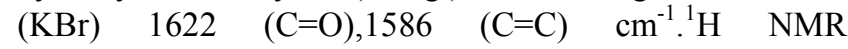
$\left(\mathrm{CDCl}_{3}\right), 214.4(\mathrm{~s}, 1 \mathrm{H}), 7.75-7.8(\mathrm{~m}, 2 \mathrm{H}), 7.22(\mathrm{~m}, 1 \mathrm{H}), 7.05(\mathrm{~s}$, $1 \mathrm{H}), 6.90(\mathrm{~m}, 1 \mathrm{H}), 6.15(\mathrm{~s}, 1 \mathrm{H}), 5.98(\mathrm{~d}, 1 \mathrm{H}, \mathrm{J}=2.26$ $\mathrm{Hz}), 5.8(\mathrm{~d}, 1 \mathrm{H}, \mathrm{J}=2.26 \mathrm{~Hz}), 3.90-4.15(\mathrm{~s}, 6 \mathrm{H}), 3.8(\mathrm{~s}$, $3 \mathrm{H})$.Yield $=46 \% ; \mathrm{mp}=73^{\circ} \mathrm{C}$; Anal. Calcd.for $\mathrm{C}_{18} \mathrm{H}_{18} \mathrm{O}_{6}: \mathrm{C}, 65.45 ; \mathrm{H}, 5.45$.found:C,65.14;H,5.57.

Compound 17: (E)-3-(2-hydroxyphenyl)-1-(2,4,6trimethoxyphenyl)prop-2-en-1-one

This compound is synthesized using 2,4,6trimethoxyacetophenone (2.10g.) and2hydroxybenzaldehyde $(1.22 \mathrm{~g}$.) as starting materials.IR(KBr)1632 $(\mathrm{C}=\mathrm{O}), 1568 \quad(\mathrm{C}=\mathrm{C}) \quad \mathrm{cm}^{-1} .{ }^{1} \mathrm{H} \quad \mathrm{NMR}$ $\left(\mathrm{CDCl}_{3}\right), 27.62(\mathrm{~d}, 1 \mathrm{HJ}=16.2 \mathrm{~Hz}), 7.42(\mathrm{~d}, 1 \mathrm{H} \mathrm{J}=8.32 \mathrm{~Hz})$, 7.18-7.34(m, 2H),6.8-6.98(m, 3H),3.85(s, 3H),3.72(s, 6H). Yield $=58 \% ; \mathrm{mp}=113-114^{\circ} \mathrm{C}$;Anal. Calcd.for $\mathrm{C}_{18} \mathrm{H}_{18} \mathrm{O}_{5}: \mathrm{C}, 68.78 ; \mathrm{H}, 5.72$;found:C,68.43;H,5.94;

Compound 18: (E)-1-(2,4,6-trimethoxyphenyl)-3-ptolylprop-2-en-1-one

This compound is synthesized using 2,4,6trimethoxyacetophenone (2.10g.) and4methylbenzaldehyde $(1.20 \mathrm{~g}$.) as starting materials.IR(KBr)1624 $(\mathrm{C}=\mathrm{O}), 1588 \quad(\mathrm{C}=\mathrm{C}) \quad \mathrm{cm}^{-1} .{ }^{1} \mathrm{H} \quad \mathrm{NMR}$ $\left(\mathrm{CDCl}_{3}\right), \partial 7.48(\mathrm{~d}, 1 \mathrm{HJ}=16.30 \mathrm{~Hz}), 7.38(\mathrm{~d}, 2 \mathrm{H} \mathrm{J}=$ $8.16 \mathrm{~Hz}), 7.17(\mathrm{~d}, 2 \mathrm{H} \mathrm{J}=8.16 \mathrm{~Hz}), 6.92(\mathrm{~d}, 1 \mathrm{H} \mathrm{J}=16.30$ $\mathrm{Hz}), 6.15(\mathrm{~d}, 2 \mathrm{H} \mathrm{J}=2.36 \mathrm{~Hz}), 3.84(\mathrm{~s}, 3 \mathrm{H}), 3.75(\mathrm{~s}, 6 \mathrm{H}), 2.32(\mathrm{~s}$, $3 \mathrm{H})$.Yield $=76 \% ; \mathrm{mp}=128-129^{\circ} \mathrm{C} ;$ Anal. Calcd.for $\mathrm{C}_{19} \mathrm{H}_{20} \mathrm{O}_{4}: \mathrm{C}, 73.07 ; \mathrm{H}, 6.41$;found:C,73.42; H,6.27.

Compound 19: 3-(4-((E)-3-(2-hydroxy-4,6dimethoxyphenyl)-3-oxoprop-1-enyl)phenyl)furan-2carbaldehyde

This compound is synthesized using 2-hydroxy-4,6'dimethoxyacetophenone (1.96g.) and2-furfuraldehyde (0.96g.) as starting materials. IR $(\mathrm{KBr}) 1626(\mathrm{C}=\mathrm{O}) .1586$ $(\mathrm{C}=\mathrm{C}) \mathrm{cm}^{-1} .{ }^{1} \mathrm{H}$ NMR $\left(\mathrm{CDCl}_{3}\right), \partial 7.79(\mathrm{~d}, 1 \mathrm{H} \mathrm{J}=15.37 \mathrm{~Hz})$, 7.57(d, $1 \mathrm{H} \mathrm{J}=15.37 \mathrm{~Hz}), 7.51(\mathrm{~s} .1 \mathrm{H}), 6.67(\mathrm{~d} .1 \mathrm{H} \mathrm{J}=3.31$ $\mathrm{Hz}), 6.5(\mathrm{t}, 1 \mathrm{H}), 3.91(\mathrm{~s} .3 \mathrm{H}), 3.83(\mathrm{~s}, 3 \mathrm{H})$. Yield $=52 \%$; $\mathrm{mp}=$ 93-94 ${ }^{0} \mathrm{C}$; Anal. Calcd.for $\mathrm{C}_{15} \mathrm{H}_{14} \mathrm{O}_{5}: \mathrm{C}, 65.69 ; \mathrm{H}, 5.1$;found:C,65.76;H,5.18.

\section{Biological Screening Methodolo- gy:Assay Method of Cytotoxic Activi- ty in Vitro}

In this study, it was aimed to evaluate the growth inhibitory effect of synthesized constituents of Aromatic chal- 
cones on human cancer cells. Acute T cell leukemia (Jurkat) and human leukemia (HL-60) cell lines were obtained from cell line bank of National Center for Cellular Sciences (NCCS), Pune, India. These cells were cultured in RPMI 1640 media containing $10 \%$ fetal bovine serum at $37^{\circ} \mathrm{C}$, $\mathrm{CO}_{2}$ incubator in the presence or absence of test compounds.Cytotoxicty was measured using the MTT [3-(4, 5dimethylthiazol-2-yl)- 2,5-diphenyl tetrasolium bromide] assay, according to the method of Mossman (1983).Briefly: the cells $(2 \times 104)$ were seeded in each well containing 0.1 $\mathrm{mL}$ of RPMI medium in 96 well plates. After $24 \mathrm{~h}$ different test concentrations ( 1 to $100 \mu \mathrm{g} / \mathrm{ml}$ ) were added and cell viability was assessed after 2 days, $10 \mu$ per well of MTT (3-(4, 5-dimethylthiazol-2-yl)-2,5-diphenyl tetrasolium bromide; $5 \mathrm{mg} / \mathrm{ml}$; stock solution, Sigma) was added to the wells. The plates were incubated at $37^{\circ} \mathrm{C}$ for additional four hours. The medium was discarded and the formazan blue, which formed in the cells, was dissolved with $100 \mu \mathrm{l}$ of DMSO. The rate of color production was measured at $570 \mathrm{~nm}$ in a spectrophotometer (Spectra MAX Plus; Molecular Devices; supported by SOFTmax PRO-3.0). All experiments were conducted under the standard laboratory illumination. The percent inhibition of cell viability was determined with reference to the control values (without test compound). The data was subjected to linear regression analysis and the regression lines were plotted for the best fit. The $\mathrm{IC}_{50}$ (inhibition of cell viability) concentrations were calculated using the respective regression equation.

\section{Results and Discussions}

Table 2 summarizes the results obtained in the assay for the inhibitory activities on human cancer cells. Cell viability assays were performed (in vitro)cytotoxic activity analyzed by MTT assays) using two human cancer cell lines.A concentration dependent decrease in cell number was observed in all tested samples demonstrated by IC50 values. This decreased cell growth in cultured human cancer cells (Jurkat and HL-60) in a concentration dependent manner is evident from table 2 and table 3 . All the analogues were compared with the basic unsubstitutedchalcone to dertermine the cytotoxic activity. The present results demonstrate that the chalcones and their analogues inhibit the proliferation of Jurkat (human T-lymphocyte leukemia) cells by triggering apoptosis.Among them the analogues 4 and 15 exhibited most potent cytotoxicity than the parental basic chalconemolecule[26]. All the test compounds have also shown cytotoxic activity (in a concentration dependent manner) against HL-60 cells [27].The most active analogues 4 and 15 on Jurkat cells exhibited activity similar to the parental molecule against HL-60.In this case, the analogues 7 and 12 have shown the more significant activity against HL-60 cells than the parental compound, but they were not more active than the parent molecule in the case of Jurkat cells. Among the synthesized chalcones, the most active compounds were 4,15 and their IC50 values on T cell leukemia (Jurkat) cells were $1.6 \times 10-2 \mathrm{mM}, 1.7 \times 10-2$
$\mathrm{mM}$ respectively.

Table 2. In vitro Cytotoxicity of chalcones and its analogues against acute T cell leukemia (Jurkat) cells by MTT assay.(Based on mM).

\begin{tabular}{|c|c|c|c|}
\hline S.No & Test compound & IC50 $(\mu \mathrm{g} / \mathrm{ml})$ & Relative Toxicity \\
\hline 1 & $\begin{array}{l}\text { Parental com } \\
\text { pound }\end{array}$ & $1.8 \times 10^{-2} \mathrm{mM}$ & 1.00 \\
\hline 2 & 4 & $1.6 \times 10^{-2} \mathrm{mM}$ & 0.88 \\
\hline 3 & 8 & $5.2 \times 10^{-2} \mathrm{mM}$ & 2.88 \\
\hline 4 & 9 & $4.1 \times 10^{-2} \mathrm{mM}$ & 2.27 \\
\hline 5 & 12 & $3.2 \times 10^{-2} \mathrm{mM}$ & 1.77 \\
\hline 6 & 14 & $4.0 \times 10^{-2} \mathrm{mM}$ & 2.22 \\
\hline 7 & 15 & $1.7 \times 10^{-2} \mathrm{mM}$ & 0.94 \\
\hline 8 & 18 & $13.1 \times 10^{-2} \mathrm{mM}$ & 7.27 \\
\hline 9 & 19 & $3.3 \times 10^{-2} \mathrm{mM}$ & 1.83 \\
\hline
\end{tabular}

[a] $\mathrm{IC}_{50}$ is defined as the concentration, which results in a $50 \%$ decrease in cell number as compared with that of the control cultures in the absence of an inhibitor. The values represent the mean $\pm \mathrm{SE}$ of four individual observation.

Table 3. In vitro Cytotoxicity of chalcones and its analogues against human leukemia (HL-60) cells by MTT assay.(Based on mM).

\begin{tabular}{|c|c|c|c|}
\hline S.No & Test compound & $\mathrm{IC}_{50}(\mu \mathrm{g} / \mathrm{ml})$ & Relative Toxicity \\
\hline 1 & $\begin{array}{l}\text { Parental } \\
\text { pound }\end{array}$ & $2.5 \times 10^{-2} \mathrm{mM}$ & 1.00 \\
\hline 2 & 4 & $2.7 \times 10^{-2} \mathrm{mM}$ & 1.08 \\
\hline 3 & 5 & $5.6 \times 10^{-2} \mathrm{mM}$ & 2.24 \\
\hline 4 & 7 & $0.47 \times 10^{-2} \mathrm{mM}$ & 0.19 \\
\hline 5 & 8 & $3.4 \times 10^{-2} \mathrm{mM}$ & 1.36 \\
\hline 6 & 9 & $2.9 \times 10^{-2} \mathrm{mM}$ & 1.16 \\
\hline 7 & 12 & $1.8 \times 10^{-2} \mathrm{mM}$ & 0.72 \\
\hline 8 & 15 & $3.4 \times 10^{-2} \mathrm{mM}$ & 1.36 \\
\hline 9 & 18 & $8.3 \times 10^{-2} \mathrm{mM}$ & 3.32 \\
\hline 10 & 19 & $4.1 \times 10^{-2} \mathrm{mM}$ & 1.64 \\
\hline
\end{tabular}

[b] $\mathrm{IC}_{50}$ is defined as the concentration, which results in a $50 \%$ decrease in cell number as compared with that of the control cultures in the absence of an inhibitor. The values represent the mean $\pm \mathrm{SE}$ of four individual observation. 


\section{Docking Studies of ChalconeAnalo- gues}

\subsection{Docking with GOLD (Genetic Optimization for Li- gand Docking)}

An attempt was made to dock the inhibitors using the superimposition of the Aromatic chalcone molecules with that of X-ray crystal structure of 1NKP for 50 times. Then, using the knowledge of the active site, an atom was defined representing the center of the active site residues, that is ARG 712 in E chain and the atom number used for docking is 2198 (taken from the protein atomic coordinates of all the active site molecules). From this point, the search was carried out in $15 \mathrm{~A} 0$ radius (since this was found to be the optimum). The annealing parameters for Vander waals and hydrogen bonding were set to $4.0 \mathrm{~A} 0$ and $2.5 \mathrm{~A} 0$ respectively. The parameters used for genetic algorithm were population size (100), selection pressure (1.1), number of operations $(1,00,000)$, number of islands $(5)$, niche size (2), migrate (10), mutate (95) and cross-over (95) (all default values). In the $1 \mathrm{NKP}$ complex, the binding interactions of the $3 \mathrm{D}$ conformation of shows that the inhibitors are located in the center of the active site, and are stabilized by Vader Waals interactions, hydrogen bonding and hydrophobic interactions. A total of 17 ligands were docked into the active site of the 1NKP site by using GOLD2.1.2 $[28,29]$.The calculated binding Gold score are given in table 4 and type of interactions in best molecules are given in table 4.The interactions of ligands with protein are maximum byVander Waals interactions. The GOLD score values are very similar in all the five top solutions. The difference between the consecutive best five runs of a particular ligand is less and this indicates the stabilization of a particular ligand at the active sites. The docking studies of the molecules are showing good fitness score and Vander Waals interactions.

Table 4. Docking scores from GOLD.

\begin{tabular}{|c|c|c|c|c|c|}
\hline Compound & Fitness & S(hb_ext) & S(vdw_ext) & $\mathrm{S}$ (hb_int) & S(vdw_int) \\
\hline Compound 1 & 28.40 & 3.04 & 25.01 & & \\
\hline Compound 2 & 27.63 & 3.39 & 25.12 & & \\
\hline Compound 3 & 26.66 & 3.13 & 26.54 & & \\
\hline Compound 4 & 29.18 & 3.43 & 26.17 & & \\
\hline Compound 5 & 28.19 & 2.95 & 25.81 & & \\
\hline Compound 6 & 25.86 & 4.36 & 24 & & \\
\hline Compound 7 & 25.76 & 3.22 & 24.52 & 0.00 & -11.18 \\
\hline
\end{tabular}

\begin{tabular}{|c|c|c|c|c|c|}
\hline Compound 8 & 28.35 & 2.91 & 26.41 & & \\
\hline Compound 10 & 26.01 & 0.00 & 27.13 & & \\
\hline Compound 11 & 25.7 & 0.00 & 25.77 & & \\
\hline Compound12 & 24.08 & 0.00 & 25.43 & & \\
\hline Compound 13 & 26 & 0.00 & 26.43 & 0.00 & 10.33 \\
\hline Compound14 & 25.96 & 0.00 & 26.33 & 0.00 & -10.24 \\
\hline Compound15 & 26.16 & 0.00 & 26.45 & & \\
\hline Compound 16 & 26.43 & 0.00 & 27.41 & 0.00 & 11.25 \\
\hline Compound 18 & 24.63 & 0.00 & 25.97 & 0.00 & -11.08 \\
\hline Compound 19 & 24.02 & 0.00 & 25.59 & 0.00 & -11.17 \\
\hline
\end{tabular}

\section{Conclusions}

To prepare new compounds with enhanced anticancer properties against human cancer cells, a series of 19 chalcone derivatives were synthesized and characterized.They were evaluated for their cytotoxicity against Jurkat (human T-lymphocyte leukemia)and HL-60 human cancer cell lines. The results demonstrate that the Aromatic chalcones and their analogues inhibit the proliferation of Jurkat (human T-lymphocyte leukemia) cells by triggering apoptosis.Among them, the analogues 4 and 15 exhibited most potent cytotoxicity than the parental molecule. The analogue 18 possessed little activity and the activity of the remaining are of intermediate range.In the case of HL-60 cells, the analogues 7 and 12 have shown more significant activity. The present study throws light on the fact that these chalcones are potent antiproliferative agents against human cancer cells without being significantly cytotoxic to normal cells. This study may lead to development of new therapeutic agents in our fight against cancer.

\section{Acknowledgement}

One of the authorsV.Srinivasarao is thankful to UGC(JRF) for providing fellowship in doing research, Principal, The Head, Department of Chemistry, University college of Science,, Saifabad, Osmania University, for providing the facilities for the work.

\section{References}

[1] C.Pouget, F. Lauthier, A. Simon, C. Fagnere, J.P.Basly, C. Delage , A.J.Chulia,Bioorg. Med. Chem. Lett.11, 309597(2001). 
[2] Y.C.Huang, J.W. Guh, Z.J.Cheng, Y.L. Chang, T.L. Hwang, C.N. Lin,C.M. Teng,Life Sci. 68, 2435-47(2001).

[3] D.V.Rosa , F. Cristiano , D. Mariagrazia, G. Cristiana , R.Antonella, B. Ezio ,M.Paolo, V. Piero, B.Federica,O.R.Franco, M.Salvatore, S.Giovanni, CancerChemother. Pharmacol. 46,305-12(2000).

[4] R.J.Anto, K.Sukumaran, G. Kuttan, M.N.A.Rao, V.Subbaraju, R. Kuttan ,Cancer Lett.97,33-37(1995).

[5] S.K.Kumar, H. Erin, P. Catherine , G. Halluru, N.E.Davidson, S.R. Khan,J. Med.Chem.46, 28132815(2003).

[6] B.L.Wei, C.H.Teng, J.P. Wang, S.J.Won, C.N. Lin, Eur. J. Med. Chem.42,660-8.( 2007).

[7] A.C.Claude, C.L. Jean, T.Patric , P. Christelle, H. Gerard, J.C.Albert, L.D.Jean, Anticancer Res. 21,3949-56(2001).

[8] R. De Vincenzo, G. Scambia, P.BenedettiPanici, F.O. Ranelletti, G.Bonanno,A. Ercoli, F.DelleMonache, F.Ferrari, M.Piantelli, S. Mancuso, Anticancer DrugDes.10, 48190(1995).

[9] E.J.Park, H.R.Park ,J.S.Lee , J.Kim, Planta Med.64, 464(1998).

[10] N.K.Parmer Sharma, M.Hussain, A.C. Watterson, J. Kumar, L.A.Samuelson,L.C. Ashok,A.K.Prasad, A. Kumar, S. Malhotra,N.Kumar, A.Jha Singh,I.Singh,Himanshu, A.Vats, N.A.Shakil , S.Trikha, S. Mukherjee,S.K. Sharma, S.K. Singh, A. Kumar, H.N.Jha, C.E. Olsen,C.P. Stove, M.E.Bracke,M.M. Mareel,Bioorg.Med. Chem.11,91329(2003).

[11] Y.M.+Lin, Y. Zhou, M.T.Flavin, L.M.Zhou, W. Nie, F.C.Chen, Bioorg. Med.Chem. 10,2795-2802(2002).

[12] S.N. Lopez, M.V. Castelli, S.A.Zacchini, J.N. Dominguez, G. Lobo, C.C. Jaime, J.C.G.Cortes, J.C. Ribas, C. Devia , M R. Ana, D.E. Ricardo Bioorg. Med. Chem. 9,19992013(2001).

[13] L.E. Alcarz, S.E.Blanco et al,Journal of theoretical Biology. 205(2),231-240,(2000).

[14] Cesar Echeverria, Oscar Donoso-Tauda, et al,International
Journal of Molecular Sciences.10,221-231(2009).

[15] Beom-Tae Kim, Kwang-JoongO,and Ki-Jun Hwang,Bull Korean ChemSoc. Vol.29, No.6,1125-1130(2008).

[16] P.Babasaheb, Bangar, A.Sachin, Patil, N. Rajesh,Bioorganic\&Medicinal ChemistryLetters. $20 \quad, 730-$ 733(2010).

[17] M.A Munawar, M Azad, H.L.Siddiqui,Journal of the Chinese Society. 55,394-400(2008).

[18] M.Azad, M.A.Munawar, H.L.Siddiqui,Journal of Applied Sciences. 7 (17),2485-2489(2007).

[19] R. Kalirazan, S.U.Sivakumar et al, International Journal of Chem TechResearch. 10,27-34,( 2009).

[20] N.Silvia, V.Lopez Maria, Castelli, A. Susana, Zacchino,.Bioorganic\&MedicinalChemistry. 9,1999-2013, (2001).

[21] Y.P.Kim , H.S.Ban, S.S. Lim, N. Kimura, S.H. Jung, J. Ji , S.Lee, N. Ryu,S.R. Keum, K.H. Shi, K.J. Obuchi, J. Pharm. Pharmacol. 53,1295-302(2001).

[22] Y. Xia, Z.Y. Yang, P. Xia, K.F.Bastow, Y.Nakanishi, K.H. Lee,Bioorg. Med.Chem.Lett 10, 699-701,(2000).

[23] J.F. Stevens, C.L. Miranda, B.Frei, D.R.Buhler, Chem. Res. Toxicol. 16,1277-86(2003).

[24] P.Boeck, C.A.BandeiraFalcao, C.P. Leal, R.A.Yunes, V.C.Filho ,E.C.Torres-Santos, B. Rossi-Bergmann, Bioorg. Med. Chem. 14,1538-45(2006).

[25] B. S. Funiss, A. J. Hannford, P. W. G. Smith, A. R. Tatchell, Vogel's Textbook of practicalorganic chemistry, fifth edition, 1032-1035(2004).

[26] Y.K. Rao, S.H. Fang, Y.M.Tzeng, Bioorg. Med. Chem.12, 2679-86(2004).

[27] H.GuraySaydam, A. Hakan,FahriSahin,Kucukoglu, E.Erciyas, E.Terzioglu,F.Buyukkececi, S.B.Omey ,Leukemia Rea. 27, 57-64(2003).

[28] G. Jones, P. Willet, R. C.Glen, J mol boil. 43,245(1995).

[29] C. peng, AvaliPy, H. B. Schegel, M. J. Frisch, J. Comp. Chem.16,49-51(1995). 